experimental models. The observed effects are partly due to iron IImediated oxidative damage. This was confirmed by the presence of a ROS scavenger delaying the onset of the effects of iron II, and crucially rendering the effects reversible upon iron-washout. These effects of tempol suggest a novel therapeutic target for the treatment of IOCM patients.

\section{A GENERIC METHOD TO ASSESS THE ADEQUACY OF INDIVIDUAL MATERNAL CARDIAC RESERVE TO TOLERATE THE DEMANDS OF PREGNANCY AND LABOUR}

doi:10.1136/heartjnl-2011-300198.173

${ }^{1} \mathrm{D}$ Barker, ${ }^{2} \mathrm{~N}$ Lewis, ${ }^{2} \mathrm{G}$ Mason, ${ }^{2} \mathrm{~L}$ B Tan. ${ }^{1}$ Liverpool Heart and Chest Hospital, Liverpool, UK; ${ }^{2}$ Leeds General Infirmary, Leeds, UK

Introduction Clinicians often feel apprehensive when managing pregnant patients with heart disease. To complement current evaluation, we have developed a new method of directly assessing the individual patient's cardiac functional reserve through stress testing. Pregnant mothers with and without heart disease were studied to test the hypothesis that pregnant cardiac patients who possess cardiac reserve equivalent to that of controls can tolerate the usual demands of pregnancy, labour and puerperium.

Methods Fifty-one pregnant women with heart disease (mean age

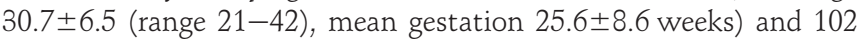
healthy pregnant women (mean age 31.4 \pm 5.0 , (range 19-41), mean gestation $25.1 \pm 9.2$ weeks) underwent maximal symptom-limited treadmill cardiopulmonary exercise testing. Fifty-nine non-pregnant women (mean age 32.7 \pm 5.1 (range 20-41) years) were similarly tested and used as a control group. Cardiac output (CO) was measured at peak exercise using the $\mathrm{CO}_{2}$ re-breathing method.
Cardiac power output (CPO) was calculated as the product of $\mathrm{CO}$ and mean arterial pressure. A composite endpoint including maternal death, fetal death, emergency caesarean section for maternal distress and significant morbidities was determined.

Results All tests were performed without significant complications. Employing data from a previous study of haemodynamics during labour in healthy women, the mean CPO required during peak labour is $2.6 \mathrm{~W}$. This value was adopted for investigation as the minimum required for an average woman to cope with the circulatory demands of normal labour. The healthy controls had a mean peak $\mathrm{CPO}(\mathrm{PkCPO})$ of $3.79 \pm 0.6 \mathrm{~W}$ and all non-pregnant women had $\mathrm{PkCPO}$ exceeding $2.6 \mathrm{~W}$. The majority of heart disease patients were able to achieve PkCPO values overlapping their healthy counterparts. Only a small proportion of the cardiac patients had $\mathrm{PkCPO}$ values lower than the $2.6 \mathrm{~W}$ cutoff. Women were significantly more likely to have uncomplicated pregnancy, labour and puerperium if able to achieve $\mathrm{PkCPO}>2.6 \mathrm{~W}$ (OR 8.1, 95\% CI 1.8 to 37.0, $\mathrm{p}=0.023$ ). Pregnant women in NYHA class I had PkCPO values indistinguishable from controls (mean $3.98 \pm 0.77 \mathrm{~W}, \mathrm{NS}$ ); whereas symptomatic pregnant women had significantly lower values (mean 3.15 $\pm 0.71 \mathrm{~W}, \mathrm{p}<0.005)$.

Conclusions Direct measurement of cardiac functional reserve capacity can be performed by maximal cardiopulmonary exercise testing with non-invasive assessment of $\mathrm{PkCPO}$, which can be safely undertaken during pregnancy. A cutoff value of PkCPO 2.6 W was identified as the lower limit for healthy women, corresponding to that required for normal labour. Most cardiac patients studied had PkCPO values comfortably above this cutoff, and all asymptomatic (NYHA I) and low risk cardiac patients had PkCPO values similar to controls. Measurement of PkCPO allows pregnant patients to be further classified into those with adequate vs limited cardiac reserve, supplementary to existing risk stratification methods. 